\title{
A vision-based system for automatic growing ring detection and measurement
}

\author{
C. Vaz ${ }^{\mathrm{a}, *}$, P. Carvalho ${ }^{\mathrm{a}}, \mathrm{F}$. Duarte $^{\mathrm{a}, \mathrm{b}, 1}$, A. Dourado ${ }^{\mathrm{a}}$

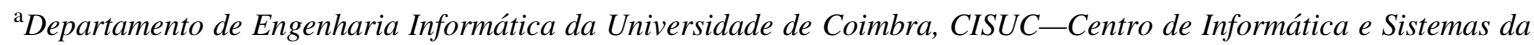 \\ Universidade de Coimbra, Pólo II, Pinhal de Marrocos, P3030 Coimbra, Portugal \\ bInstituto Superior de Engenharia de Coimbra, Quinta da Nora, Apartado 10057, P3031 Coimbra, Portugal
}

\begin{abstract}
An automatic visual inspection system for wood growing ring identification and measurement is described. Given the irregular contrast distributions in wood samples, the identification of these elements is not straightforward. Furthermore, it is observed that many rings exhibit very narrow widths. To overcome these problems, several new methods are introduced, namely at the local image scale definition and at the image segmentation levels. First, local scales are automatically computed and applied for ring center identification. This information is then used to reconstruct the noisy image signal, which is segmented using a local minimum distance-minimum cross-entropy principle. The system uses a reduced and intuitive set of user defined parameters; for this reason, it is easy to tune.
\end{abstract}

(C) 2004 Elsevier Ltd. All rights reserved.

Keywords: Automatic visual inspection and measurement; Automatic scale definition; Adaptive image segmentation; Cross-entropy

\section{Introduction}

Forestry, ecological and botanical researchers spend much of their time measuring annual ring data for their analysis of forest growth and dynamics. One of their methods of sample collection is to cut down the tree and then to cut one or more cross-sectional discs from the tree's trunk. Once these samples have been collected, they must be prepared for analysis. At the very least, this preparation includes sanding to remove marks left by the saw and may also include polishing. Once a sample has been prepared, there are a number of devices that can potentially help the researchers to gather information of

\footnotetext{
* Corresponding author.

E-mail addresses: cmvaz@dei.uc.pt (C. Vaz), carvalho@dei.uc.pt (P. Carvalho), fduarte@dei.uc.pt (F. Duarte), dourado@ dei.uc.pt (A. Dourado).

${ }^{1}$ Tel.: + 351-239790000; fax: +351-239701266.
} 
interest. Some of these devices are purely mechanical, many include electronics and a few include a computer. All of these devices require the operator to decide where one ring ends and the next begins. The measurement systems that involve computers are currently at what has been called the 'third generation' of development. The main characteristics of these third generation systems is the use of a high resolution input device (scanner or camera) to digitize an image and the ability to save results to disk. Processing the image then requires an operator to identify regions or features in the image, after which the computer attempts to use this information either to count the features or to identify and then count the features in the image. In addition to eliminating human effort, there are many benefits to be gained by letting the computer decide where tree ring boundaries are located. These benefits can be described as follows: (i) it may take less time to analyze each sample, assuming an efficient algorithm and/or a fast computer; (ii) the results of analyzing a given sample would be repeatable and; (iii) more accurate information would result.

In this paper, an automatic measurement system for tree growing rings that does not require user intervention (except for the introduction of the radial analysis direction) for ring identification and measurement is described. Due to the low contrast between growing rings and the noise present in these types of images, our tests revealed that it is not possible to rely directly on standard segmentation algorithms. Therefore, it was necessary to develop new methods in order to solve this specific problem. In our approach, ring centers are first identified using a scale-space representation of the image. This representation enables automatic selection of local scales, which, in turn, permits robust identification of the ring centers. This method is described in Section 2.1. After center identification, the system proceeds with ring border computation using a minimum distance-minimum cross-entropy principle, which is introduced in Sections 2.2 and 2.3. Finally, in Section 3, some main conclusions and the results obtained with this inspection system are presented.

\section{Identification of growing rings}

Most wood species exhibit a low contrast between growing rings, in several cases being of the order of the CCD's noise characteristic. Furthermore, significant spatial variations in contrast usually occur in wood samples. It is also observed that most latewood rings exhibit very small widths (several rings exhibit widths between 3 and 4 pixels in the digital image). These characteristics impose severe constraints upon image segmentation, which is a very critical operation since it affects the shapes of the identified objects and, therefore, greatly influences the inspection system's accuracy. To avoid noise influence during the segmentation of the image, false maximums and minimums are detected and the image signal between adjacent noisy pixels is reconstructed by constraining the signal as a type $C^{1}$ function. This method enables subpixel accuracy, which turns out to be of major importance, given the small widths of several rings. Using the reconstructed signal, a local minimum distance-minimum cross-entropy principal is applied to identify ring transitions.

\subsection{Growing rings detection with automatic scale selection}

We consider the black growing rings as line-like structures with a slight curvature. It is assumed that the medulla center of the sample is known a priori. Hence, the ring direction at each point can be estimated as being perpendicular to the radius that passes through each ring. The gray profile across the longitudinal 
direction shows a dip on the contrast between the line ring structure and the adjacent white rings that can be considered background, while the gray-value profile along the longitudinal direction shows only slight variations. The gray-value profile across the ring can be considered to be a generalized edge with a Gaussian profile. Since the typical profile is known and the orientation can be estimated, an optimal operator for ring detection is constructed based on a unidirectional approach along the radial direction. However, the scale of each ring is not know a priori, so an operator must be applied for different scales in order to identify the scale leading to the strongest response. In this work, we use a multi-scale detector approach, based on the idea of normalized derivatives introduced by Lindeberg (1993).

The dependency of the second derivatives with width $\sigma$ of a Gaussian kernel $g(\mathbf{x}, \sigma)$ applied to a line structure with a Gaussian gray-value profile $f(\mathbf{x})$ across the line is applied to estimate the width of the structure. In the framework of the classical scale space theory, Lindeberg (1993) suggests the use of local extrema (with respect to $\sigma$ ) of $\gamma$-parameterized normalized derivatives of the form $\partial / \partial_{\mathbf{x}, \gamma-\text { norm }}=\sigma^{\gamma} \partial / \partial_{\mathbf{x}}$-for scale estimation. The general principle of scale selection states that scale levels for feature detection can be selected from scales where normalized differential invariants assume maxima over the scales. Taking up this idea for line structures, we look for a normalized second derivative. The line response function is defined as $r(\mathbf{x}, \sigma)$

$$
r(\mathbf{x}, \sigma)=\sigma^{\gamma} \frac{\mathrm{d}^{2} h(\mathbf{x}, \sigma)}{\mathrm{d} \mathbf{x}^{2}}
$$

with $h(\mathbf{x}, \sigma)=f(\mathbf{x}) \otimes g(\mathbf{x}, \sigma)$ being the convolution of the line profile $f(\mathbf{x})$ obtained in the radial direction near the ring and the Gaussian kernel of width $\sigma$. Lorentz, Carlsen, Buzug, Fassnacht, and Weese (1997) have shown that (i) for a line with a Gaussian profile of width $\sigma_{0}$ and unitary contrast, convolved with a Gaussian of width $\sigma$, yields a Gaussian of width $\sigma_{\text {tot }}=\sqrt{\sigma_{0}^{2}+\sigma^{2}}$ and (ii) to obtain $\sigma_{\text {opt }}=\sigma_{0}, \gamma$ must be $3 / 2$, leading to $r\left(\mathbf{0}, \sigma_{\text {opt }}\right)=-1 / \sqrt{8 \sigma_{\mathrm{opt}}}$. This framework presents good stability for features detection. Fig. 1 shows the results of the space scale extrema (i.e. points that are extrema both in space and scale) of the line response function obtained along a radial direction. Note the ability of the method to extract each one of the black rings.

\subsection{Signal reconstruction}

Let $\Pi=\left[\mathbf{x}_{1}, \ldots, \mathbf{x}_{n}\right]$ be the coordinates of the points that define the radial section introduced by the user, $\Gamma=\left[\mathbf{x}_{1}, \ldots, \mathbf{x}_{n}\right]$ be the coordinates of the estimated ring centers detected with the line detector

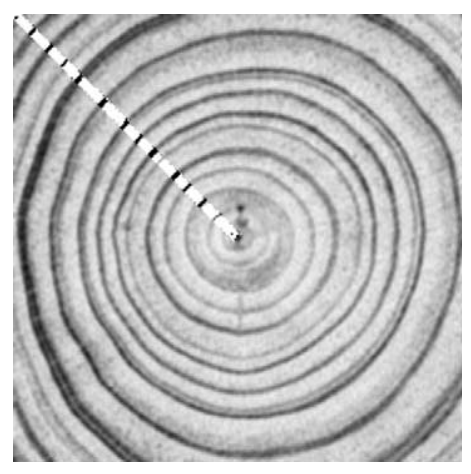

Fig. 1. Multi-scale blob detection using normalized scale-space extrema of the squared Laplacian. 
along $\prod$, as introduced in Section 2.1, and $f(\mathbf{x}), \mathbf{x} \in \Pi$ be the gray values of the image along the given section. As the acquired image exhibits the CCD's noise, as a first step, the algorithm estimates a noise-free function $\hat{f}(\mathbf{x})$. For this purpose, all false maximums and minimums of $f(\mathbf{x})$ are identified. Let $\aleph$ be the group of minimums and maximums of $f(\mathbf{x})$ and let $F(\mathbf{x})$ be defined by $F\left(\mathbf{x}_{i}\right)=f\left(\mathbf{x}_{i}\right)-f\left(\mathbf{x}_{i+1}\right)$. Then $\boldsymbol{\aleph}$ is defined as in Eq. (2)

$$
\begin{aligned}
\aleph=\{ & \left\{\mathbf{x}_{i} \in \prod: F\left(\mathbf{x}_{i}\right) F\left(\mathbf{x}_{i+1}\right)<0\right\} \bigcup\left\{\mathbf{x}_{j}=\frac{\mathbf{x}_{i}+\mathbf{x}_{k}}{2} \in \prod: F\left(\mathbf{x}_{i-1}\right) F\left(\mathbf{x}_{k}\right)<0 \wedge f\left(\mathbf{x}_{i}\right)\right. \\
& \left.-f\left(\mathbf{x}_{z}\right)=0, \mathbf{x}_{i}, \mathbf{x}_{k}, \mathbf{x}_{z} \in \prod, z=i+1 \ldots k\right\}
\end{aligned}
$$

While the first part of the definition in Eq. (2) is due to sharp local inflections of $f(\mathbf{x})$, the second part is for local inflections identification where maxima or minima extend for several points.

Clearly, all points in $\aleph$, except those that correspond to the ring centers, identify noisy data segments. To estimate the noise-free signal, it is assumed that it exhibits a monotonously decreasing behavior between earlywood and latewood ring centers and monotonously increasing behavior between latewood and earlywood ring centers. Hence, for each pair of points $\mathbf{x}_{i}, \mathbf{x}_{i+1} \in \mathcal{K}$, which verify the constrain $\mathbf{x}_{i}, \mathbf{x}_{i+1} \notin \Gamma$, i.e. are not ring centers, the signal has to be reconstructed. For this purpose, it is assumed that the signal is of type $C^{1}$ with a maximum of one inflection in the noisy segment. We apply a third-order polynomial $\hat{f}(\mathbf{x})=a x^{3}+b x^{2}+c x+d$ approach to reconstruct the noise information. Namely, the curve is reconstructed using an interpolation obtained with the minimization of the following (note that this formulation corresponds to a $C^{1}$ curve):

$$
\begin{aligned}
& E=\left\|\left[\begin{array}{llll}
3\left|\mathbf{w}_{i}\right|^{2} & 2\left|\mathbf{w}_{i}\right| & 1 & 0 \\
3\left|\mathbf{w}_{i+2}\right|^{2} & 3\left|\mathbf{w}_{i+2}\right|^{2} & 1 & 0 \\
\left|\mathbf{w}_{i}\right|^{3} & \left|\mathbf{w}_{i}\right|^{2} & \left|\mathbf{w}_{i}\right| & 1 \\
\left|\mathbf{w}_{i+2}\right|^{3} & \left|\mathbf{w}_{i+2}\right|^{2} & \left|\mathbf{w}_{i+2}\right| & 1
\end{array}\right]\left[\begin{array}{l}
a \\
b \\
c \\
d
\end{array}\right]-\left[\begin{array}{c}
\frac{\mathrm{d} f}{\mathrm{~d} \mathbf{x}}\left(\mathbf{w}_{i}\right) \\
\frac{\mathrm{d} f}{\mathrm{~d} \mathbf{x}}\left(\mathbf{w}_{i+2}\right) \\
f\left(\mathbf{w}_{i}\right) \\
f\left(\mathbf{w}_{i+2}\right)
\end{array}\right]\right\|^{2}, \\
& \left\{\begin{array}{l}
\mathbf{w}_{j}=\frac{\mathbf{x}_{j-1}+\mathbf{x}_{j}}{2}, \\
\mathbf{x}_{i-1}, \mathbf{x}_{i}, \mathbf{x}_{i+1}, \mathbf{x}_{i+2} \in \aleph
\end{array}\right.
\end{aligned}
$$

This procedure is shown in Fig. 2 and in Fig. 3 some approximation results are shown.

\subsection{Local minimum distance-minimum cross-entropy ring identification}

After signal reconstruction, the system proceeds with a segmentation phase, i.e. the system identifies the transitions between earlywood and latewood rings. This is performed with a cross-entropy similarity measure. Ideally, transitions between rings should induce rapid changes in the curve's slopes. However, given the low contrast that characterizes most of the wood samples, it is observed that it is almost impossible to distinguish these transition regions from the ring's interior regions. 


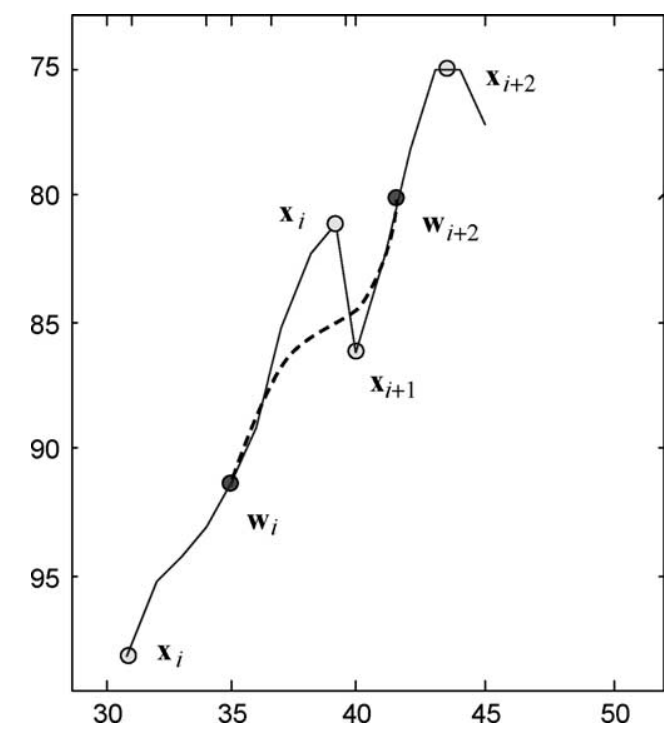

Fig. 2. Approximation procedure. Original curve—continuous; approximated curve dotted.

Therefore, the main idea at this stage for each segment comprehended between adjacent ring centers is to partition the curve into two homogenous subsegments with maximum resemblance to the original curve.

Let $\mathfrak{J}: \mathbb{R}_{+} \times \mathbb{R}_{+} \rightarrow \mathbb{R}_{+}$and $\mu_{1}, \mu_{2} \in \mathbb{R}_{+}$be the partition function such that

$$
\mathfrak{J}(y, T) \triangleq\left\{\begin{array}{l}
\mu_{1} \Leftarrow y<T \\
\mu_{2} \Leftarrow y \geq T
\end{array}\right.
$$

and let $S: \mathbb{R}_{+} \times \mathbb{R}_{+}^{N} \rightarrow \mathbb{R}$ be the similarity measure between $f(\mathbf{x})$ and $\mathfrak{I}(f(\mathbf{x}), T)$. There are several possible definitions for $S$. For instance, in Brink (1989) the correlation between $f(\mathbf{x})$ and $\mathfrak{J}(f(\mathbf{x}), T)$ is used, while in Kapur, Sahoo, and Wong, 1985, Li and Lee, 1993, Pal and Bhandari, 1993, and Shoo,

(a)

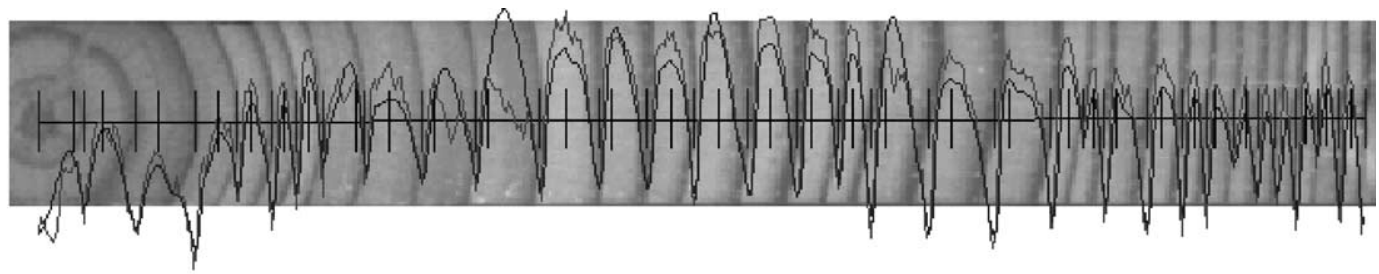

(b)

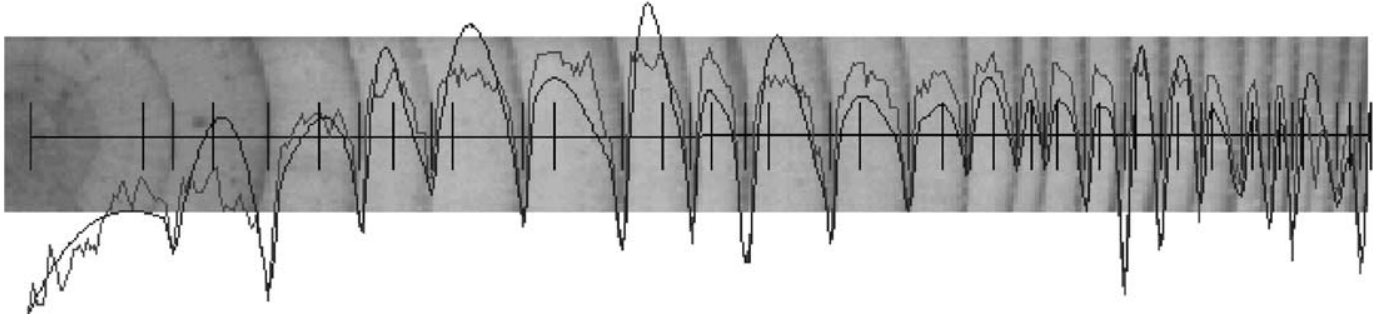

Fig. 3. Estimation results of $\hat{f}(\mathbf{x})$. 
Soltani, Wong, and Chen, (1988), maximum entropy is applied. For this particular problem, it is observed that the best similarity measure is the cross-entropy measure, i.e. let $f(\mathbf{x}) \in\left[y_{m}, y_{M}\right]$ and $\operatorname{Pr}(f(\mathbf{x})=y)$ be the probability of occurrence of $f(\mathbf{x})=y$ for the segment under consideration:

$$
S(T, f(\mathbf{x}))=\int_{y_{m}}^{T} \operatorname{Pr}(f(\mathbf{x})=y) \log _{2}\left(\frac{\operatorname{Pr}(f(\mathbf{x})=y)}{\operatorname{Pr}\left(\mu_{1}\right)}\right) \mathrm{d} y+\int_{T}^{y_{M}} \operatorname{Pr}(f(\mathbf{x})=y) \log _{2}\left(\frac{\operatorname{Pr}(f(\mathbf{x})=y)}{\operatorname{Pr}\left(\mu_{2}\right)}\right) \mathrm{d} y
$$

Given that latewood rings exhibit very small widths, subpixel resolution is highly important, since an error of one pixel during width identification may lead to substantial biased measurements. To avoid this, $f(\mathbf{x})$ and $\hat{f}(\mathbf{x})$ are further linearly interpolated using a step of $\Delta_{1}$ (we use $\Delta_{1}=0.1$ ) between consecutive points. Using this approach, it can be shown that Eq. (5) can be rewritten by

$$
\begin{aligned}
S(T)= & \sum_{i=1}^{T-1} H(i)\left(y_{m}+\Delta(i-0.5)\right) \log _{2}\left(\frac{H(i)\left(y_{m}+\Delta(i-0.5)\right)}{\mu_{1}}\right)+\sum_{i=T}^{N} H(i)\left(y_{m}+\Delta(i-0.5)\right) \\
& \times \log _{2}\left(\frac{H(i)\left(y_{m}+\Delta(i-0.5)\right)}{\mu_{2}}\right)
\end{aligned}
$$

where $N=\left\lceil y_{M}-y_{m} / \Delta\right\rceil, \Delta$, number of discretization levels (we use $\Delta=100$ )

$$
\mu_{1}=\frac{\sum_{i=1}^{T-1} H(i)\left(y_{m}+\Delta(i-0.5)\right)}{\sum_{i=1}^{T-1} H(i)}, \quad \mu_{2}=\frac{\sum_{i=T}^{N} H(i)\left(y_{m}+\Delta(i-0.5)\right)}{\sum_{i=T}^{N} H(i)}
$$

$H(i)$, number of points such that $\left.\left.f(\mathbf{x})-y_{m} \in\right] \Delta(i-1), \Delta i\right]$

Using Eq. (6), the curve is partitioned such that $\mathfrak{J}\left(y, T^{*}\right)$, where

$$
S\left(T^{*}, f(\mathbf{x})\right)=\min _{T \in] y_{m}, y_{M}[}\{S(T, f(\mathbf{x}))\}
$$

The transition between rings is selected at point $\mathbf{x}^{*}$, such that $f\left(\mathbf{x}^{*}\right) \leq T^{*} \wedge f\left(\mathbf{x}^{*}+\varepsilon\right)>T^{*}, \varepsilon \rightarrow O^{+}$.

Eq. (7) is applied with $f(\mathbf{x})$ and $\hat{f}(\mathbf{x})$. Let $\mathbf{x}^{*}$ and $\hat{\mathbf{x}}^{*}$, be the transition points obtained with $f(\mathbf{x})$ and $\hat{f}(\mathbf{x})$, respectively, and let $\mathbf{x}_{m}$ be the coordinates of the latewood ring center under consideration. The actual transition point is obtained from:

$$
\mathbf{x}=\left\{\begin{array}{l}
\mathbf{x}^{*} \Leftarrow\left|\mathbf{x}^{*}-\mathbf{x}_{m}\right|<\left|\hat{\mathbf{x}}^{*}-\mathbf{x}_{m}\right| \\
\hat{\mathbf{x}}^{*} \Leftarrow\left|\mathbf{x}^{*}-\mathbf{x}_{m}\right| \geq\left|\hat{\mathbf{x}}^{*}-\mathbf{x}_{m}\right|
\end{array}\right.
$$

Usually, it is observed that Eq. (7) produces exact results when applied to $\hat{f}(\mathbf{x})$ since $f(\mathbf{x})$ may lead to identifications of $T^{*}$ which correspond to local minimums that are more pronounced inside the brighter rings (note that CCD noise increases quadratically with the intensity level (Healey \& Kondepudy, 1991)). However, it is also observed that Eq. (3) may lead to estimations of $\hat{f}(\mathbf{x})$ that are too smooth, leading, therefore, to biased estimations of $T^{*}$. These smooth estimations will occur whenever the data between centers exhibit large concentrations of noise. In these cases, it is observed that points $\mathbf{w}$ will be chosen near the centers and, therefore, exhibit small slopes. Hence, to minimize these effects-given that latewood rings are much smaller than earlywood rings-Eq. (8) is applied. 


\section{Results and conclusions}

An automatic system for tree growing rings detection and measurement is introduced. First, ring centers are identified using a scale-space representation of the image. The purposed algorithm is able to identify each optimal local scale autonomously, i.e. it enables the extraction of the meaningful features in the image (in this case, the ring centers), avoiding false centers detection, which otherwise could occur due to noise interference. After ring center identification, the system proceeds with ring border computation, using a minimum distance-minimum cross-entropy principle. This is a two-step procedure: (i) first, the image along the radial analysis direction is reconstructed using a 'low-pass filtering' approach with cubic polynomials; and (ii) a maximal resemblance measure, computed upon minimum cross-entropy, is applied to map gray levels into two classes. This procedure has proven to be very robust.

Theoretically, the developed method does not require any user intervention, except for the definition of the radial direction for ring analysis. However, due to the computational load imposed by the optimal scale selection procedure described in Section 2.1, the operator should specify the range of admissible scales by providing a minimum and a maximum limit for the search interval. These are the only parameters required by the system. It is observed that their definition can be provided once and used for all subsequent inspections. The only reason for avoiding very large search interval specification is due to the added computational load.

The described algorithms have been integrated in an automatic visual inspection system. Fig. 4 shows the results of the analysis of some Pinus pinaster (pine wood) samples and in Fig. 5, the result obtained in the analysis of a cross-section disc sample is depicted. As can be observed, the method correctly detects the tree ring boundaries without user intervention, even for images with very low contrast and very narrow rings, as in Fig. 4c, and is able to detect false rings, as can be observed in Fig. 5.

This system is currently being used in several national sylviculture research projects under development. In comparison with traditional hand-performed inspections, the proposed system enables much faster and more accurate sample analysis. Namely, it is usually observed that hand-performed inspections take up to two hours per sample, while with this new approach, an inspection can be

(a)

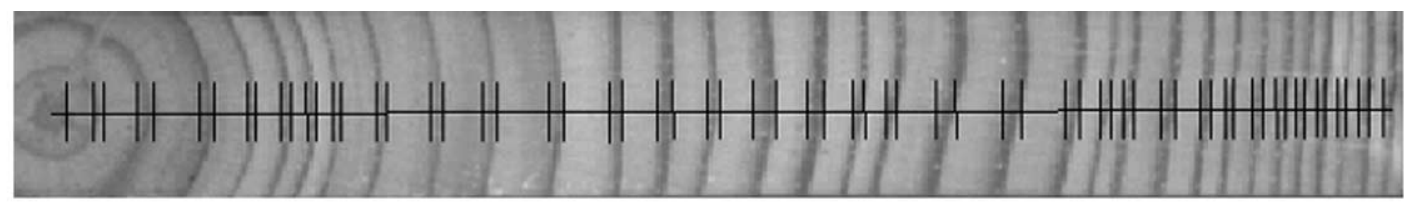

(b)

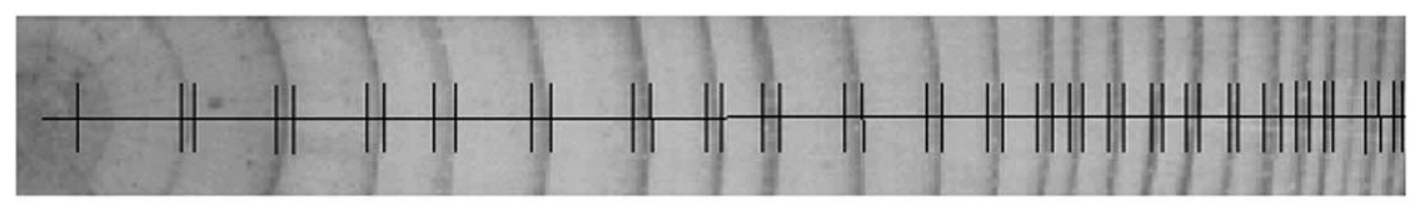

(c)

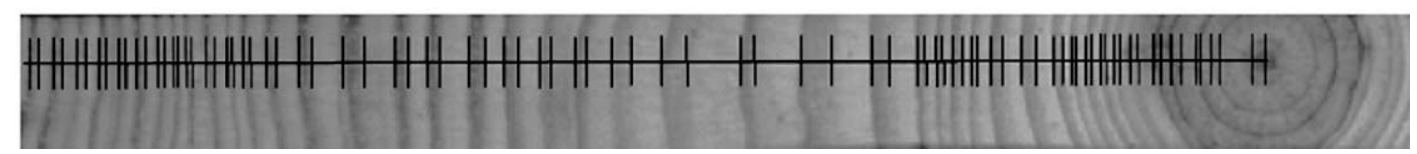

Fig. 4. Results obtained by the outline method for some Pinus pinaster samples. 


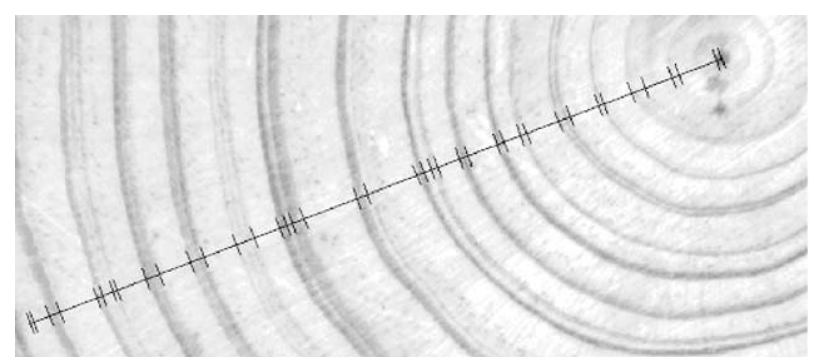

Fig. 5. Result obtained by the outline method for a cross-section disc sample.

conducted in less than two minutes if a $1 \mathrm{GHz}$ Pentium III computer is applied. As for accuracy, results reported with hand-made measurements have less than $1 / 3 \mathrm{~mm}$, while this system is able to achieve much better accuracies, which, nevertheless, are dependant on the applied zoom factor of the lens.

\section{Acknowledgements}

This project was partially funded by the PAMAF and by FCT/FCTUC/PRAXIS XXI, and was performed at the Computational Perception Laboratory at CISUC.

\section{References}

Brink, A. D. (1989). Grey-level thresholding of images using a correlation criterion. Pattern Recognition Letters, 9(5), $335-341$.

Healey, G., \& Kondepudy, R. (1991). Modeling and calibrating CCD cameras for illumination insensitive machine vision. Proceedings of the SPIE \#1614 conference on optics, illumination, and image sensing for machine vision VI, SPIE, pp. $121-132$.

Kapur, J. N., Sahoo, P. K., \& Wong, A. K. C. (1985). A new method for gray-scale picture thresholding using entropy of histogram. Computer Vision, Graphical Models and Image Processing, 29, 273-285.

Li, C. H., \& Lee, C. K. (1993). Minimum cross entropy thresholding. Pattern Recognition, 26(4), 617-625.

Lindeberg, T. (1993). On scale selection for differential operators. Proceedings of the eighth Scandinavian conference on image analysis, Tromso, Norway, pp. 857-866.

Lorentz, C., Carlsen, I. C., Buzug, T. M., Fassnacht, C., \& Weese, J. (1997). A multi-scale line filter with automatic scale selection based on the Hessian matrix for medical image segmentation. Proceedings of the first international conference, scale-space'97, Ultrecht, Netherlands, pp. 152-163.

Pal, N. R., \& Bhandari, D. (1993). Image thresholding: some new techniques. Signal Processing, 33, 139-158.

Shoo, P. K., Soltani, S., Wong, A. K. C., \& Chen, T. C. (1988). A survey of threshold techniques. Computer Vision, Graphics and Image Processing, 41. 